\title{
NURBS Method for Cycloidal Steel Ball Reducer Profile Design
}

\author{
Wang Juanjuan $^{1,2}$, Ping Xueliang ${ }^{1, *}$ \\ ${ }^{1}$ School of Mechanical Engineering, Jiangnan University, China \\ ${ }^{2}$ Jiangsu Province Key Laboratory of Advanced Food Manufacturing Equipment and Technology, Jiangnan University, China
}

Copyright $\bigcirc 2019$ by authors, all rights reserved. Authors agree that this article remains permanently open access under the terms of the Creative Commons Attribution License 4.0 International License

\begin{abstract}
The profile design of cycloid disk in cycloidal steel ball reducer is the core of reducer design, which directly affects the performance of reducer. The cycloidal ball reducer is driven by a certain number of steel balls in a tooth profile composed of a pair of intermeshing internal and external cycloidal lines. The performance of the reducer can be optimized by adjusting the design equation of the cycloidal disc type line. However, in the process of design and optimization, the requirement of freedom and controllability of cycloid is getting higher and higher. The traditional design method of cycloidal profile based on cycloid parameter equation can no longer meet the requirements of design. The NURBS method can accurately represent the free curve and the conic quadratic curve. The cycloidal profile is actually a complex curve composed of a certain number of arcs. In this paper, the NURBS curve is applied to the design of cycloid disk of reducer, and the cycloidal profile based on NURBS is deeply studied. By sampling the known cycloidal profile, the sample point, the inverse control point, the weight factor, the node vector and the sample point are obtained, and the analysis verifies that the error between the fitted NURBS curve and the original curve is within the feasible range. Taking the BR85us-10G-6 reducer as an example, the fitting curve of the inner cycloid disk profile is obtained by using cubic NURBS curve, which can improve the flexibility and local adjustment of the cycloidal disc profile design.
\end{abstract}

Keywords Cycloidal Steel Ball Reducer, Profile Design, Non-uniform Rational B-spline, Curve Fitting

\section{Introduction}

Reducer is an important part of industrial production, especially in industrial robot industry. The innovation and development of reducer is the key to the progress of robot industry in the future. Cycloidal steel ball reducer is a kind of cycloidal meshing high-end precision transmission device. It has the advantages of large transmission ratio, compact structure, high transmission efficiency and especially without backlash. It is used in aerospace, robot, machine tool and so on. Military industry has higher requirements for transmission accuracy of the device [1]. Cycloidal steel ball planetary transmission mechanism is essentially a kind of two-tooth difference $\mathrm{K}-\mathrm{H}-\mathrm{V}$ planetary transmission device. This deceleration drive mechanism transmits motion and power through steel ball, and the tooth profile of steel ball motion is formed by meshing the inner and outer cycloids [2]. The deceleration drive is accomplished by the continuous rotation of the steel ball in the tooth profile $[3,4]$. Design calculation method steps, transmission characteristics and principle, cycloid equation [5], transmission ratio calculation, parameter selection, force and strength analysis [6], dynamic balance [7] and vibration analysis [8], CAD/CAM and other aspects of research have achieved a lot of research results, and there are few studies on the design of the profile of the cycloidal disc.

The cycloidal disc is one of the important components of the cycloidal ball reducer, and the performance of the reducer is directly affected by its cycloidal disc profile. The design parameters of the cycloidal disc are closely related to whether the cycloidal groove is undercut, and the efficiency and load bearing of the reducer are significant. Cai Feng et al [9], analyzed in detail the influence of the value of short-amplitude coefficient on the transmission performance of the steel ball reducer, and studied the range of the short-amplitude coefficient. Wang Lixing [10] and Xi Qingkun [11] et al. optimized the efficiency, volume and bearing of the steel ball reducer based on genetic algorithm. The research optimized the design parameters of the steel ball reducer to some extent. At present, the design method of drawing the inner and outer cycloidal disc profile according to the cycloid equation and the given condition parameters is widely used in the design of the cycloidal disc profile, but the overall profile will be changed when the design parameters of the profile is 
modified, avoiding the undercutting while reducing the efficiency and carrying capacity, the optimization of the overall performance of the reducer cannot be achieved, so the introduction of the NURBS curve design method to achieve a partial adjustment of the cycloidal disc profile. The NURBS curve design method has been widely used in various types of profile design and free curve design [12]. In the design of screw rotor profile, Shyh Hau [13] proposed to use the cubic spline curve to fit the point on the original rotor tool profile line to obtain the screw type. Line; Long Ji et al [14] studied the continuity in the design of NURBS curves and improved the curvature stability of the profile design; He Xueming et al [15] use the straight line, arc, and cubic B-spline curve for the reverse design of the yin-yang rotor for the meshing line segment. The NURBS curve design method provides a new idea for the design of the cycloidal disc profile. On the basis of the original profile design, the subsequent partial optimization is performed by the NURBS representation profile, which can improve the flexibility of the line design.

\section{Forward Design Principle of Cycloid Steel Ball Reducer Profile}

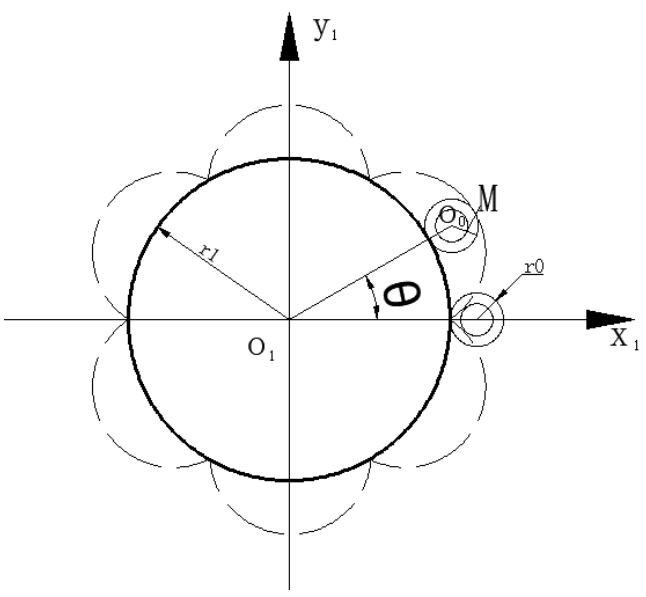

Figure 1. Outer cycloid forming principle diagram

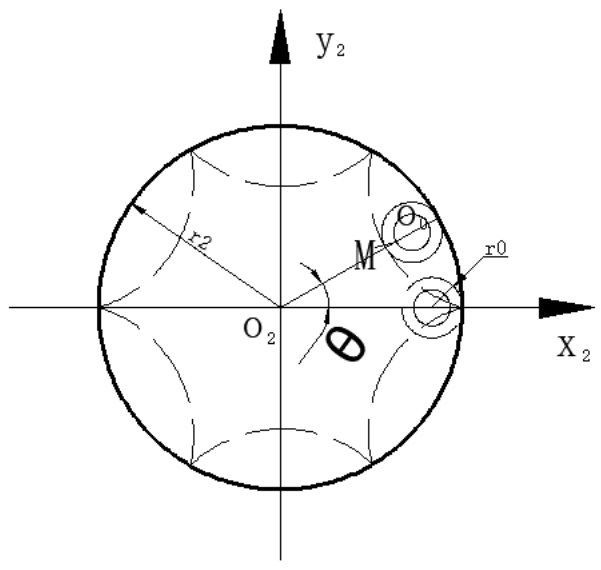

Figure 2. Inner cycloid forming principle diagram
The theoretical tooth profile curve of deceleration mechanism is a pair of meshing inner and outer cycloid of short amplitude. The forming method of inner and outer cycloid of short amplitude includes two kinds of methods: enclosing method and non-enveloping method. The equation of inner and outer cycloid is deduced according to the non-enveloping method as an example. Figure 1 shows a schematic diagram of cycloid generation. In that figure, The center of a rolling circle " $\mathrm{O}_{0}$ " rolls purely around the center of a base circle " $O_{1}$ ", $M$ is the point in the circle and the eccentricity, $\mathrm{e}=\mathrm{O}_{0} M$. The number of teeth generated from the outer cycloid is defined as Eq. (1), the motion track of the $M$ point is the outer cycloid. Figure 2 shows the generating principle diagram of inner cycloid. In the figure 2, The center of a rolling circle " $O_{0}$ " rolls purely around the center of a base circle " $\mathrm{O}_{2} ", M$ ' is the inner point of the circle, the eccentricity, $A=O_{0} M^{\prime}$. The number of teeth generated by the inner cycloid is defined as Eq. (2), and the motion track of the $M$ ' point is the inner cycloid.

$$
\begin{aligned}
& \mathrm{Z}_{1}=\mathrm{R}_{1} / \mathrm{R}_{0} \\
& \mathrm{Z}_{2}=\mathrm{R}_{2} / \mathrm{R}_{0}
\end{aligned}
$$

$Z_{1}$-- The number of teeth generated from the outer cycloid.

$R_{1}$-- The radius of the outer cycloid base circle.

$R_{2}$-- The radius of the inner cycloid base circle.

$R_{0}$-- The radius of the base circle.

$Z_{2}$-- The number of teeth generated by the inner cycloid.

The inner cycloid equation is as follows:

$$
\left\{\begin{array}{l}
X=d_{s} \times \cos \theta+c \times \cos \left(n_{b} \times \theta\right) \\
Y=d_{s} \times \sin \theta-c \times \sin \left(n_{b} \times \theta\right)
\end{array}\right.
$$

The outer cycloid equation is as follows:

$$
\left\{\begin{array}{l}
X=d_{s} \times \cos \theta-c \times \cos \left(n_{b} \times \theta\right) \\
Y=d_{s} \times \sin \theta-c \times \sin \left(n_{b} \times \theta\right)
\end{array}\right.
$$

$d_{s}$ is the circle diameter of the steel ball distribution, and its calculation equation is as follows:

$$
d_{s}=r_{d} / \sin \left(180 / n_{h}\right)
$$

$r_{d}$-- Radius of the steel ball.

$\theta$-- The angle at which the base circle is rolled when the unwrapped circle is rolled purely on the base circle.

$c$-- Half of the eccentricity.

$n_{b}$-- Number of steel balls.

Referring to the traditional forward design method of cycloidal steel ball reducer, the parameter design process of cycloidal steel ball reducer mainly includes the following steps. The main calculation formulas for the forward design are shown in Table 1. 
Table 1. Cycloid Design Formula

\begin{tabular}{|c|c|c|}
\hline Name & Symbol & Calculation formula \\
\hline Number of steel balls & $n_{b}$ & Determined by transmission ratio \\
\hline Eccentricity & $A$ & Transfer power and model \\
\hline Diameter of steel ball & $d_{b}$ & Transfer power and model \\
\hline Radius of base circle of epicycloid & $r_{e}$ & $r_{e}=R_{b} n_{e} / n_{b}$ \\
\hline Radius of base circle of hypocycloid & $r_{h}$ & $r_{h}=R_{b} n_{h} / n_{b}$ \\
\hline Radius of roll & $r_{0}$ & $r_{0}=R_{b} / n_{b}$ \\
\hline Distribution radius of steel ball & $r_{s}$ & $r_{s}=n_{b} r_{0}$ \\
\hline Wave number of epicycloid & $n_{e}$ & $n_{e}=n_{b}-1$ \\
\hline Wave number of hypocycloid & $n_{h}$ & $n_{h}=n_{b}+1$ \\
\hline Short amplitude coefficient & $K_{b}$ & $k n_{b} / 2 R_{b}$ \\
\hline Spherical diameter coefficient & $K_{2}$ & $K_{2}=d_{z} \sin \left(\pi / n_{b}\right) / d_{b}$ \\
\hline
\end{tabular}

(1) Select the reduction ratio.

(2) Determine the number of steel balls based on the reduction ratio, input speed, and input power.

(3) Determine the number of inner and outer cycloidal waves by determining the relationship between the number of inner and outer cycloidal waves and the number of steel balls determined according to the meshing principle.

(4) Select the eccentricity and the standard steel ball diameter in the series table.

(5) Calculate the basic parameters of the epicycloid and hypocycloid.

\section{NURBS Curve Theory in Profile Design}

NURBS curve is a kind of non-uniform rational B-spline curve with excellent smoothness and controllability, and its highly free curve characteristics greatly increase the flexibility of the curve construction. The one-time NURBS curve has the following definition.

$$
C(u)=\frac{\sum_{i=0}^{n} N_{i, p(u)} \omega_{i} P_{i}}{\sum_{i=0}^{n} N_{i, p(u)} \omega_{i}}, \quad a \leq u \leq b
$$

$\left\{P_{i}\right\}$-- The control point.

$\left\{\omega_{i}\right\}$-- The weight factor of the control point which determines the degree of control points from the curve.

$\left\{N_{i, p(u)}\right\}$-- A p times B-spline basis function defined on the aperiodic node vector $\mathrm{U}$.

A set of node vectors $u \in\left[u_{0}, u_{1}, \ldots u_{n+p+1}\right]$ satisfies the non-decreasing sequence of nodes. Let $s$ be the repeatability of two endpoint nodes, then $s \leq p+1$. Let $s^{\prime}$ be the repeatability of inner nodes, then $s^{\prime} \leq p$. The non-uniform B-spline basis functions are defined by such a set of node vectors. In order to control the characteristics at the end of NURBS curve, the definition domain of NURBS curve is generally taken as $u \in\left[u_{p}, u_{n+1}\right]=[0,1]$, where $u_{0}=u_{1}, \ldots,=u_{p}=0$ and $u_{n+1}=u_{n+2}, \ldots,=u_{n+p+1}=1$. The inner nodes $u_{p+1}, u_{p+2}, \ldots, u_{n}$ are determined according to the calculation method of node vectors.

The B-spline basis function can be defined by the difference quotient definition of the truncated power function, the definition of flowering, and the recursion formula proposed by Debour, Cox and Mansfield, etc. This paper uses the recursive definition method, as follows:

$$
\begin{gathered}
N_{i, 0(u)}= \begin{cases}1, & \text { if } u_{\mathrm{i}} \leq u<u_{i+1} \\
0, & \text { others }\end{cases} \\
N_{i, p(u)}=\frac{u-u_{\mathrm{i}}}{u_{i+p}-u_{\mathrm{i}}} N_{i, p-1(u)}+\frac{u_{i+p+1}-u}{u_{i+p+1}-u_{\mathrm{i}+1}} N_{i+1, p-1(u)}
\end{gathered}
$$

$u_{\mathrm{i}}$-- node.

$N_{i, 0(u)}$-- A step function which is equal to zero outside the half-open interval $u \in\left[u_{\mathrm{i}}, u_{i+1}\right)$.

$N_{i, p(u)}{ }^{--}$A linear combination of two $p-1$ times basis functions when $p>0$.

According to the above calculation method, a NURBS profile can be obtained by defining the control point and the corresponding weight factor of the profile and calculating the node vector and the basis function.

\section{NURBS Profile Design Method}

\subsection{Getting Profile Data of BR85us-10G-6}

It can be seen from the design of the reducer profile that directly expressing the profile of the reducer with the 
NUBRS curve firstly requires obtaining the data of the original profile, and using the control points and weight factor of the profile, the endpoint of the profile is used as the node of the NURBS curve. The cumulative chord length method is used to calculate the node vector of the entire section line, making it a one-stage reducer type profile. In the process of NURBS curve profile design, it is necessary to control the vectors of the endpoints of each segment, and then the designed profile can achieve first-order continuity. Taking the model BR85us-10G-6 reducer as an example, the cubic spline curve interpolation is used to design the reducer. The cubic spline curve is used because it can reach the minimum number of consecutive $\mathrm{G}^{2}$. The profile data of the reducer prototype are obtained. The design process is shown in Figure 3.

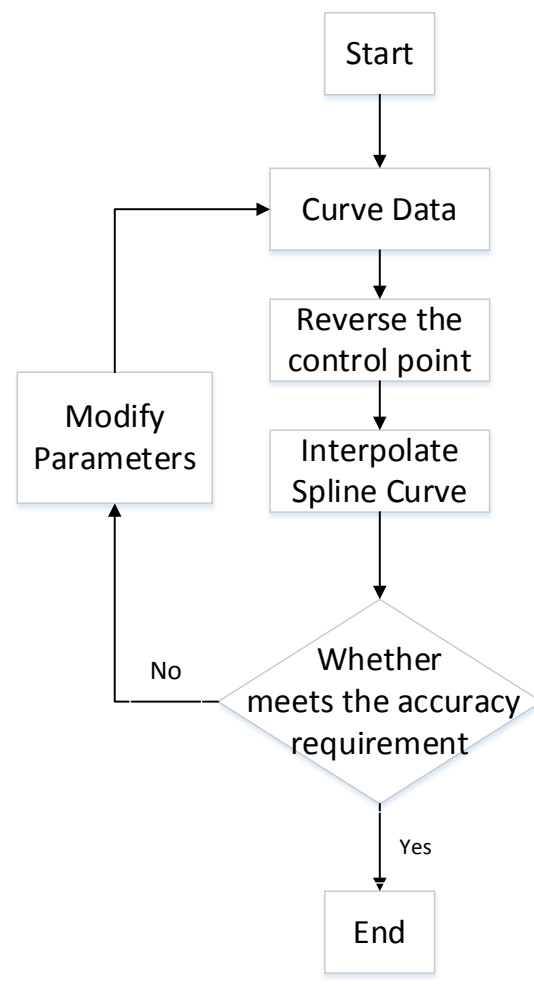

Figure 3. NURBS curve design process

The design method of cycloidal disc profile of reducer is the same as that of inner cycloidal disc. Taking the inner cycloidal disc profile as an example, the design of outer cycloidal disc profile can be completed by referring to the design process of inner cycloidal disc profile. The design parameters of the cycloidal disk profile of BR85us-10G-6 reducer are shown in the Table 2. According to the parameter equation, the cycloidal disk profile of the reducer can be obtained as shown in Figure 4.

Table 2. Cycloidal disk profile design parameters

\begin{tabular}{|c|c|c|c|c|}
\hline $\begin{array}{c}\text { Number } \\
\text { of steel } \\
\text { balls }\end{array}$ & $\begin{array}{c}\text { Eccentricity } \\
{[\mathrm{mm}]}\end{array}$ & $\begin{array}{c}\text { Diameter } \\
\text { of steel } \\
\text { ball[mm] }\end{array}$ & $\begin{array}{c}\text { Short } \\
\text { amplitude } \\
\text { coefficient }\end{array}$ & $\begin{array}{c}\text { Radius } \\
\text { of roll } \\
\text { [mm] }\end{array}$ \\
\hline 21 & 2 & 3.5 & 0.89425 & 1.11825 \\
\hline
\end{tabular}

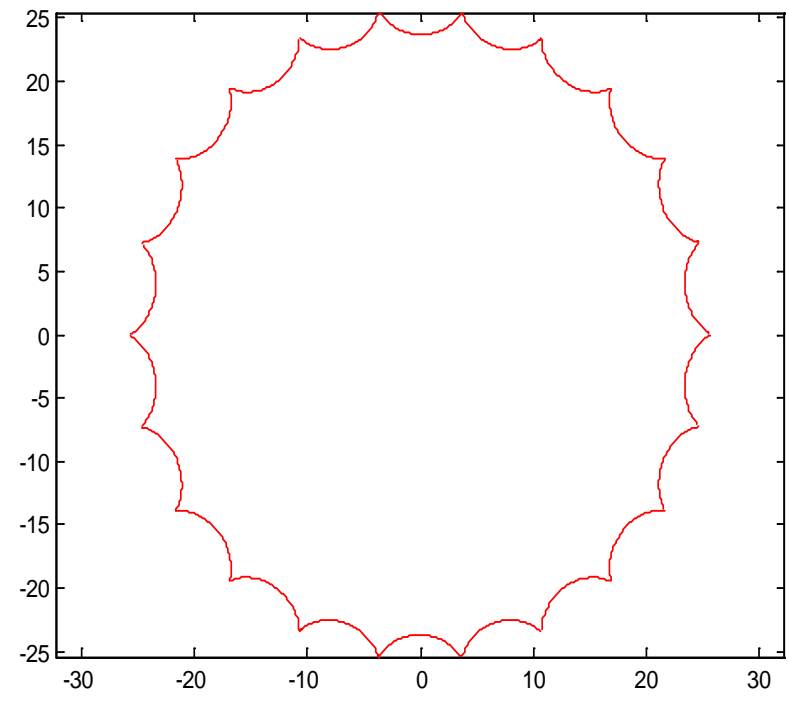

Figure 4. BR85us-10G-6 cycloidal disk profile

According to the geometric characteristics of the inner cycloid disk profile, a complete profile is connected by the same curve of $\mathrm{n}$ segments, in which $\mathrm{n}$ is determined by the deceleration ratio of the reducer. In order to simplify the fitting process, one segment is fitted, and then the other segments are fitted and linked into the whole profile with the same design method. Because the curvature of the inner cycloid disc varies greatly, the sampling method and sampling points have great influence on the accuracy of curve fitting design. Different sampling methods and NURBS of different control points are used to design the inner cycloid disk profile.

\subsection{Node Vector Determination of Cycloidal Disk Profile Data Points}

The parameterization of data points is an important step in curve construction and plays an important role in curve fairness. In order to obtain better smoothness, the cumulative chord length parameter method can be used to parameterize the data points of profiles.

$\mathrm{L}$ is the total length of the curve. $L=\sum_{i=1}^{n}\left|Q_{i}-Q_{i-1}\right|$, when $\mathrm{u}_{0}=0 \quad \mathrm{u}_{\mathrm{n}}=1, \quad \mathrm{u}_{\mathrm{i}}=\mathrm{u}_{\mathrm{i}-1}+\frac{\left|Q_{i}-Q_{i-1}\right|}{L}, \quad \mathrm{i}=1, \ldots, \mathrm{n}-1$. This parameterization truly reflects the distribution of data points according to chord length. The interpolation curve obtained has good fairness and has been widely used.

\subsection{Inversely Find the Control Vertex of NURBS Interpolation Curve}

When the cubic NURBS curve is used to express a series of data points on the measured cycloidal disk profile, the control points of the NURBS curve must be inversely calculated according to the node vectors determined by the type points on the curve. 
Using the same processing method as non-uniform B-spline curve inversion, the control points are calculated by using the type value points as data points. The weights of all control points are first taken as 1 . After the control points are obtained, the curve is adjusted according to the corresponding weights.

The cubic non-uniform B-spline curve equation for interpolating $n+1$ data points is as follows:

$$
\mathrm{C}(u)=\sum_{j=i}^{i+3} P_{j} N_{j, 3}(u) \quad \mathrm{u} \in\left[u_{i+3}, u_{i+4}\right] \subset\left[u_{3}, u_{n+3}\right]
$$

Substituting $Q_{i}$ and its corresponding node values into the upper formula, a set of linear equations composed of $\mathrm{n}+1$ vector equations is obtained, as follows:

$$
\begin{gathered}
\mathrm{C}\left(u_{i+3}\right)=\sum_{j=i}^{i+3} P_{j} N_{j, 3}\left(u_{i+3}\right)=P_{i} N_{i, 3}\left(u_{i+3}\right)+P_{i+1} N_{i+1,3}\left(u_{i+3}\right)+P_{i+2} N_{i+2,3}\left(u_{i+3}\right)+P_{i+3} N_{i+3,3}\left(u_{i+3}\right)=Q_{i} \\
\mathrm{u} \in\left[u_{i+3}, u_{i+4}\right] \subset\left[u_{3}, u_{n+3}\right] \quad \mathrm{i}=0,1, \ldots, \mathrm{n}
\end{gathered}
$$

Additionally, two additional equations given by boundary conditions are added.

$$
\left\{\begin{array}{l}
P_{1}-P_{0}=\frac{u_{4}-u_{3}}{3} C_{0}^{\prime} \\
P_{n+2}-P_{n+1}=\frac{u_{n+3}-u_{n+2}}{3} C_{n}^{\prime}
\end{array}\right.
$$

$C_{0}^{\prime}$-- The tangent vectors at the left endpoints.

$C_{n}^{\prime}$-- The tangent vectors at the right endpoints.

$$
\begin{aligned}
& {\left[\begin{array}{c}
1 \\
N_{0,3}\left(u_{3}\right)
\end{array}\right.} \\
& -1 \\
& N_{1,3}\left(u_{3}\right) \quad N_{2,3}\left(u_{3}\right) \quad N_{3,3}\left(u_{3}\right) \\
& N_{1,3}\left(u_{4}\right) \quad N_{2,3}\left(u_{4}\right) \quad N_{3,3}\left(u_{4}\right) \quad N_{4,3}\left(u_{4}\right) \\
& \ddots \quad \ddots \\
& \begin{array}{cc}
\ddots & \ddots \\
N_{n-1,3}\left(u_{n+2}\right) & N_{n, 3}\left(u_{n+2}\right)
\end{array} \\
& N_{n-1,3}\left(u_{n+3}\right) \\
& N_{n, 3}\left(u_{n+3}\right) \\
& N_{n+1,3}\left(u_{n+2}\right) \\
& N_{n+2,3}\left(u_{n+2}\right) \\
& N_{n+1,3}\left(u_{n+3}\right) \\
& N_{n+2,3}\left(u_{n+3}\right) \\
& 1 \\
& -1]\left[\begin{array}{c}
n+1 \\
P_{n+2}
\end{array}\right] \\
& {\left[\begin{array}{c}
P_{0} \\
P_{1} \\
P_{2} \\
\vdots \\
P_{n} \\
P_{n+1} \\
P_{n+2}
\end{array}\right]=\left[\begin{array}{l}
a \\
Q_{0} \\
Q_{1} \\
\vdots \\
Q_{n-1} \\
Q_{n} \\
b
\end{array}\right]} \\
& a=\frac{u_{4}-u_{3}}{3} C_{0}^{\prime} \quad, b=\frac{u_{n+3}-u_{n+2}}{3} C_{n}^{\prime}
\end{aligned}
$$

$Q_{n}{ }^{--}$The data point value.

\subsection{Fitting Result}

Figure 5 and figure 7 adopt two different sampling methods respectively. Figure 6 is the local enlargement of the right and left ends of the fitting curve obtained by the first sampling method. Because the curvature at the left and right ends changes rapidly, the fitting curve deviates greatly from the original curve. In order to avoid the influence caused by the rapid change of curvature at the fitting end point, the sampling method shown in figure 7 is adopted to make the curve at both ends smooth and increase the sampling frequency in the middle section, so as to reduce the influence of the rapid change of curvature on the trend of the fitting curve. 


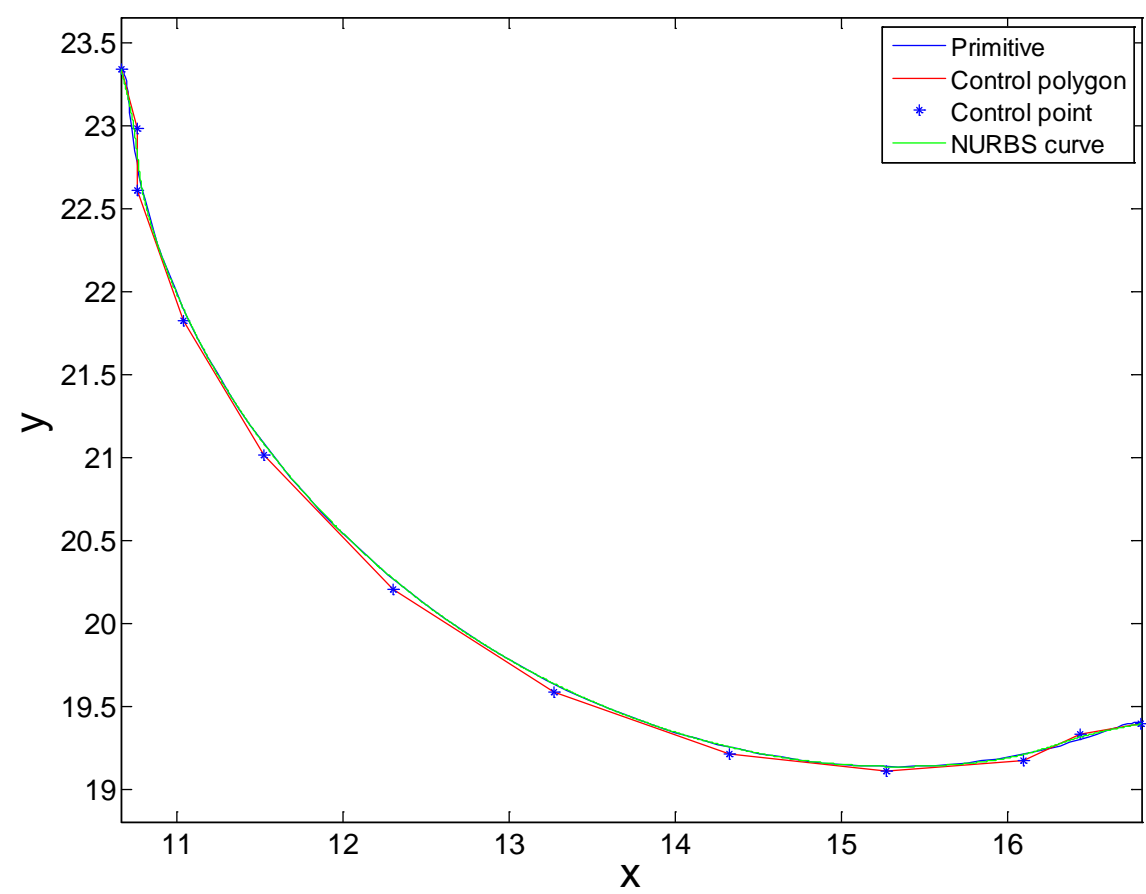

Figure 5. The first type of NURBS curve fitting profile

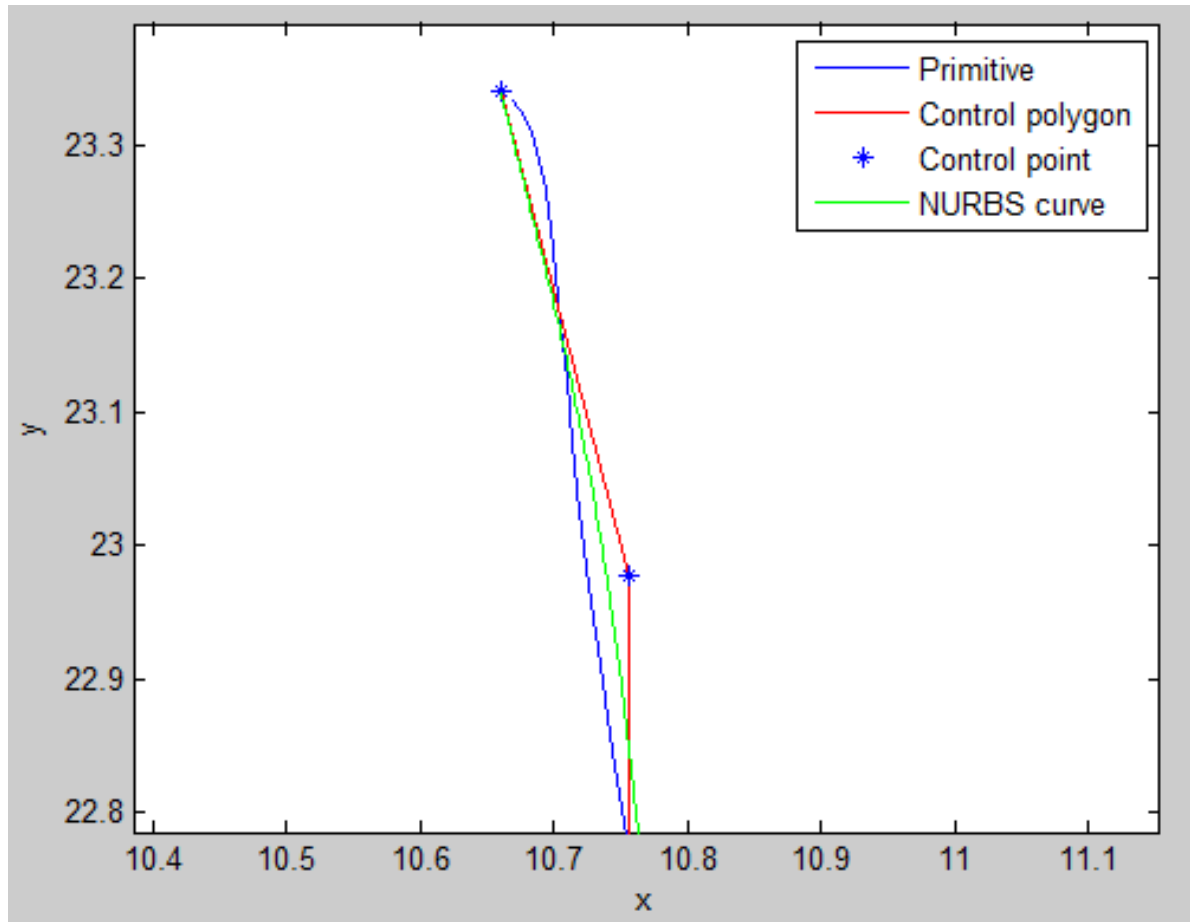

(a) Partial enlargement of the left end 


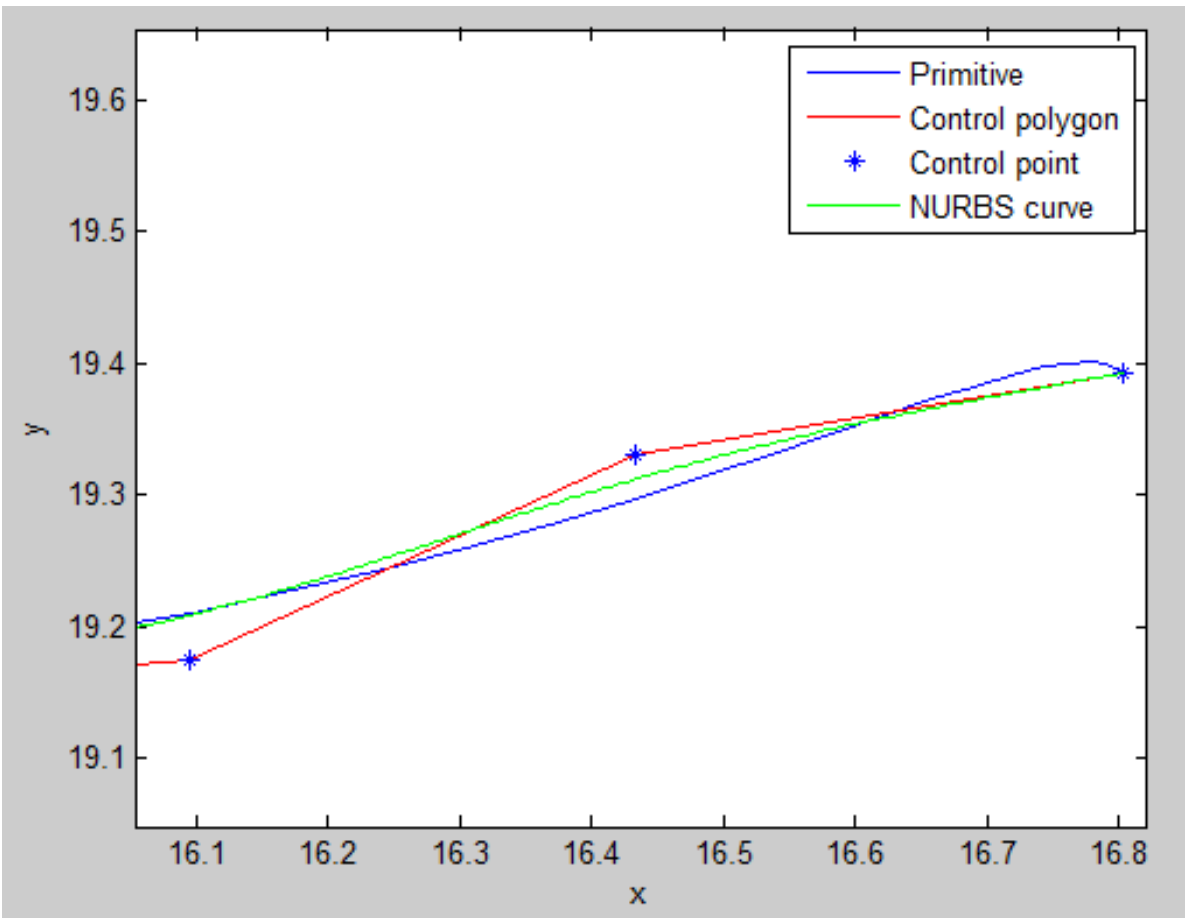

(b) Partial enlargement of the right end

Figure 6. Local enlargement of NURBS curve fitting profile

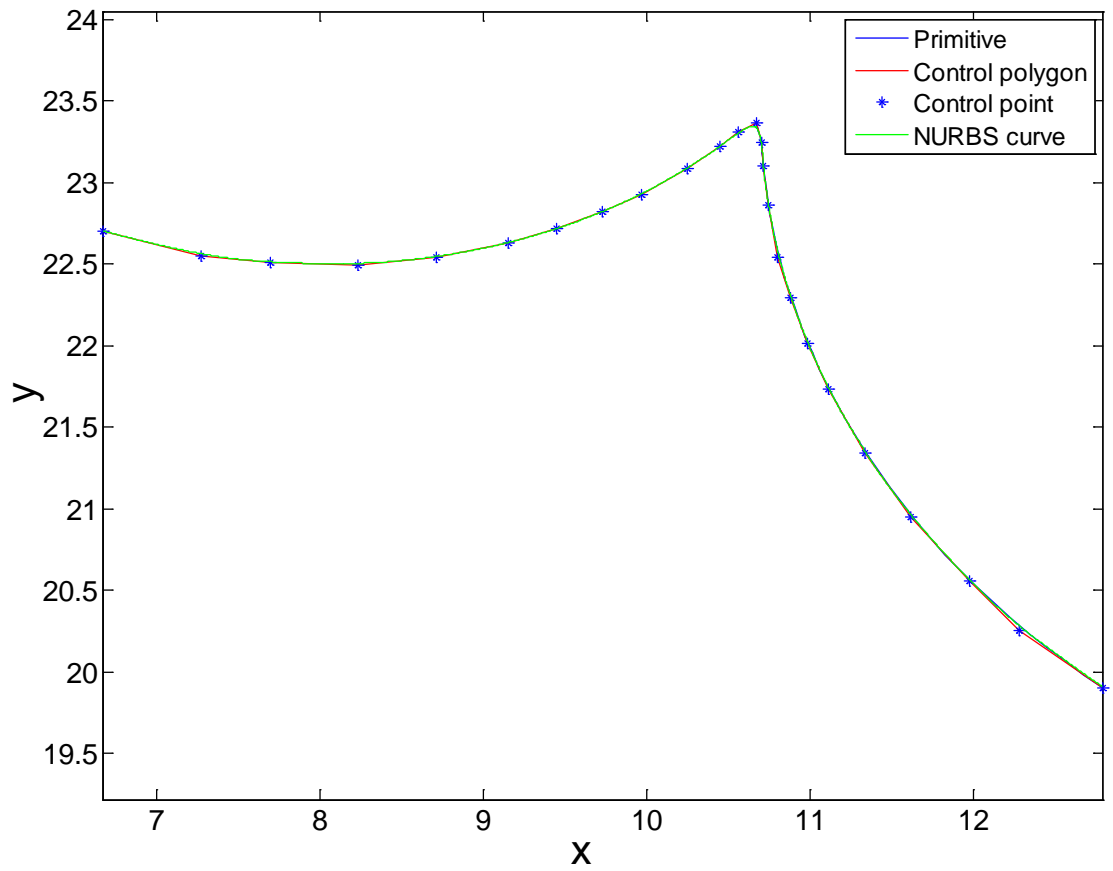

Figure 7. The second type of NURBS curve fitting profile

Figure 8 shows the complete cycloidal disk profile obtained by using NURBS profile design method. 


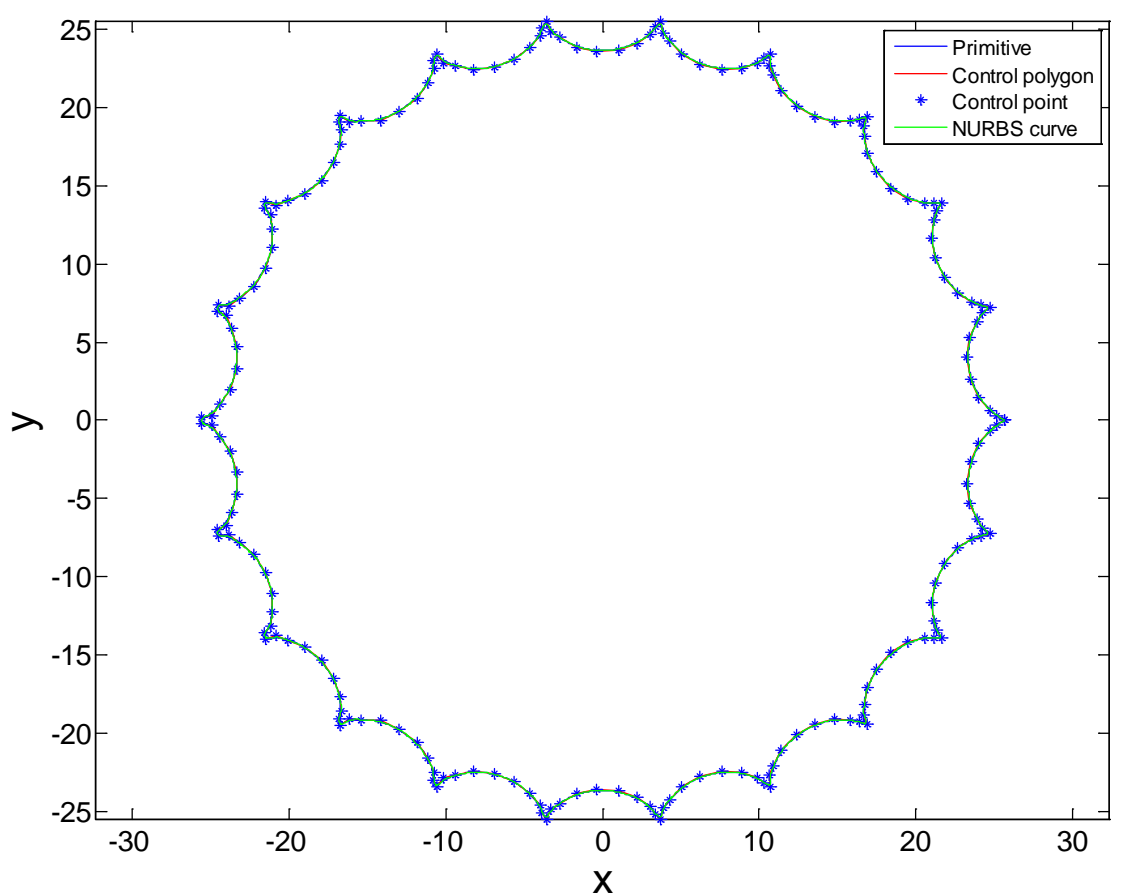

Figure 8. NURBS curve fitting of the whole shape of the cycloid

\subsection{Error Analysis of Fitting Accuracy}

The basic method for calculating fitting error between fitting curve and data points is to calculate the distance between data points and curves. Average distance and maximum distance are the main evaluation indexes. In order to better compare the errors of various fitting methods, two fitting curve error analysis methods are used to analyze the accuracy error of the fitting results of the last stage.

Sum of Squares due to Error (SSE):

$$
S S E=\sum_{i=1}^{n} \omega_{i}\left(y_{i}-\hat{y}_{i}\right)^{2}
$$

The statistical parameter calculates the sum of the error squares of the corresponding points between the fitting data and the original data. The closer SSE is to 0 , the better model selection and fitting are, and the more successful data prediction is.

Sum of Squares for total (SST):

$$
S S T=\sum_{i=1}^{n} \omega_{i}\left(y_{i}-\bar{y}_{i}\right)^{2}
$$

$$
\text { R-square }=1-\frac{S S E}{S S T}
$$

$n$-- Sample size.

$\omega_{i}-$ - The weight factor of data.

$y_{i}$-- The value of sample data.

$\hat{y}_{i}$-- The average of sample data.

R-square represents the fitness of a fitting by the change of data. The normal range of determined coefficients is [0, $1]$, and the closer to 1 , it shows that the stronger the explanatory power of the variables of the equation to $y$, the better the model fits the data.

As shown in figure 9, error 1 is the fitting accuracy error of the fitting curve obtained by the first sampling method, error 2 is the fitting accuracy error of the fitting curve obtained by the second sampling method, and error 3 is the fitting accuracy error of the fitting curve obtained by increasing the number of control points on the basis of the second sampling method. 


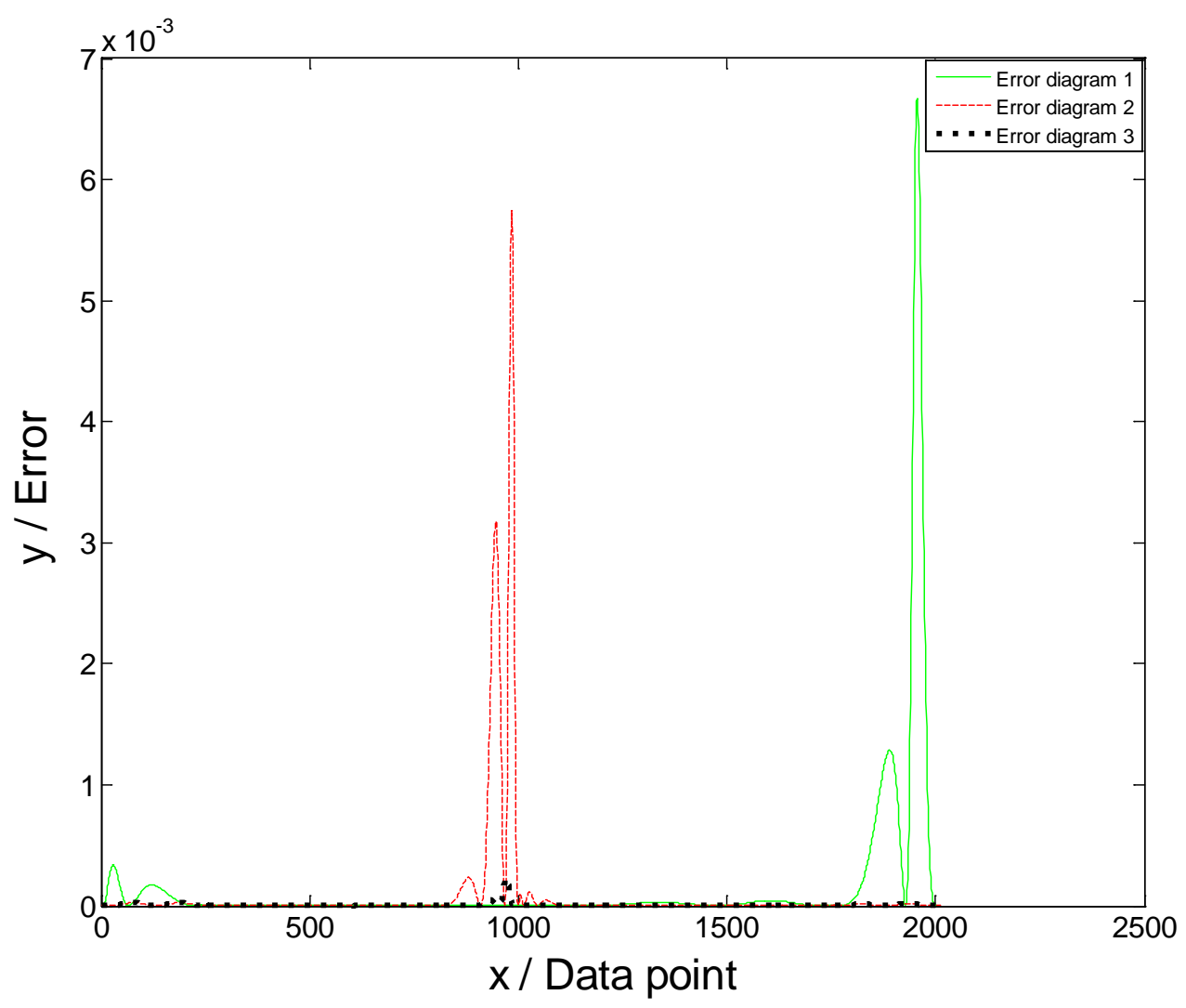

Figure 9. Error diagram of NURBS curve fitting profile

\section{Conclusions}

For the optimization design process of the cycloidal disc, the relative optimization of the objective function is achieved by adjusting the values of each parameter within a certain range, and the optimal value of each target is not obtained. The degree of freedom of the traditional rule curve cannot satisfy the problem of contour design flexibility. A method for optimizing and redesigning traditional rule curves using NURBS curves is proposed. By sampling the known cycloidal contours, the sampling points, inverse control points, weighting factors, node vectors and sampling points are obtained, and the analysis is verified. The error between the fitted NURBS curve and the original curve is within the feasible range to obtain the cycloidal contour with higher degree of freedom and curve continuity. The profile expressed by the method can not only accurately represent the cycloidal profile features, but also has excellent local modification characteristics.

The design method of NURBS profiles is proposed, and the design of the cycloidal profile of the reducer prototype is carried out with 3 NURBS curves. The cycloidal disk profiles with a precision error of 0.1 micron from the original profiles are designed by this method. According to the local controllability of NURBS curve, the cycloidal disk profile can be modified locally. The local characteristics of the cycloidal disc profile can be improved without affecting the performance of other parts, thereby optimizing the cycloidal disc profile and further optimizing the performance of the reducer.

\section{Acknowledgements}

We are very grateful to the experts for their comments and suggestions for this paper, and we would like to thank the "Postgraduate Research \& Practice Innovation Program of Jiangsu Provincial” for funding.

\section{REFERENCES}

[1] Yao Sufen, Gao Dongqiang, Li Jin. Three-Dimensional Modeling Technology of Double Cycloidal Steel Ball Reducer Based on UG, J. Journal of Shaanxi University of Science and Technology, 2008, 26(6):138-141.

[2] Gao Dongqiang, Yao Sufen, Li Jin. Research on Parametric Design Technology of Double Cycloidal Steel Ball Reducer Based on PRO/E, J. Mechanical Design \& Manufacturing, 2009(3):222-224.

[3] Terada H.The Development of gearless reducers with rolling balls, J. Journal of Mechanical Science \& Technology, 2010, 24(1):189-195.

[4] H . Terada, R. Sueki, K. Makino, T. Mitsuishi. Development of an Outer Groove Rotor Type Precession 
Motion Ball Reducer. The 14th IFToMM World congress, Taipei, Taiwan, October 25-30,2015

[5] Ren Xiuzhi. Design of Cycloidal Steel Ball Reducer and Research on Cycloidal Disc Processing Method,D.

[6] An Zijun, Yang Ronggang, Yi Yali. Meshing normal force and elastic hysteresis of precision steel ball transmission, $\mathrm{J}$. Journal of Mechanical Engineering, 2016, 52(9): 42-48

[7] Yang Ronggang, An Zijun. Research on Nonlinear Dynamic Characteristics of Cycloid Ball Planetary Transmission Constant Velocity Output Mechanism Based on Harmonic Balance Method, J. Journal of Vibration and Shock, 2017, 36(2):153-158.

[8] Zhang Peng,An Zijun. Dynamic Modeling and Inherent Characteristic Analysis of Cycloid Ball Planetary Transmission, J.China Mechanical Engineering, 2014, 25(2):157-162.

[9] CAI Feng, ZHANG Peng, ZHANG Yu-hua, et al. Research on Value Method of Short-Amplitude Coefficient in Cycloid Ball Planetary Transmission, J. Mechanical Transmission, 2014(3):27-30.

[10] Wang Lixing, Zhu Junping, Qin Yupei, et al. Optimization Design of Cycloid Ball Planetary Reducer Based on Genetic Algorithm, J. Journal of Agricultural Mechanization Research, 2011, 33(4).

[11] Xi Qingkun. Optimization Design of Cycloid Ball Planetary Reducer Based on Genetic Algorithm, J. Mechanical Transmission, 2011, 35(3): 24-26.

[12] Chen Shaoping. Interpolation and Application of Cubic NURBS Curves, J. Mechanical Science and Technology, 2001, 20(5): 692-693.

[13] Su S H, Tseng C H. Generating conjugate shapes using piecewise cubic spline functions, J. Computer Methods in Applied Mechanics \& Engineering, 2000, 187(1):245-260.

[14] Huang Hainan, He Xueming, Zhang Rong, et al. Inverse design of screw rotor profile based on NURBS curve, J. Food and Machinery, 2018, v.34;No.197(03):98-102+150.

[15] Long Wei, He Xueming, Wu Jia, et al. Research on Curve Continuity in Forward Design of Screw Rotor Profiles, J. Mechanical Science and Technology, 2019(2):190-197. 